\title{
Limit Groups of Equationally Noetherian Groups
}

\author{
Abderezak OULD HOUCINE
}

\begin{abstract}
We collect in this paper some remarks and observations about limit groups of equationally noetherian groups. We show in particular, that some known properties of limit groups of a free group or, more generally, of a torsion-free hyperbolic group can be seen as consequences of the fact that such groups are equationally noetherian. Especially, such properties are still true for linear groups and finitely generated abelian-by-nilpotent groups.
\end{abstract}

\section{Introduction}

Limit groups of free groups have been introduced by Sela [20] for his study of equations over free groups. They can be seen, geometrically and algebraically, as limits of free groups. This class coincides with the class of fully residually-free groups; a class of groups introduced by Baumslag [1] and studied by Kharlampovich and Myasnikov [12, 13, 14], Remeslennikov [14], Sela [20] and by many others $[5,9,11,19]$.

Some properties of limit groups, as the stationarity of any sequence of epimorphisms, can be deduced from the linearity of free groups. In fact they are consequences of the noetherian nature of the Zariski topology of the field of complex numbers; that is any system of equations in a free group is equivalent to a finite subsystem [8]. This last property is very interesting and a group satisfying it is called equationally noetherian; a notion introduced by Baumslag, Myasnikov and Remeslennikov [2]. The purpose of this paper is to see that some known properties of limit groups of free groups or of a torsion-free hyperbolic group can be seen as consequences of the fact such groups are equationally noetherian. These properties are still true for limit groups of any equationally noetherian group and in particular of a linear group and a finitely generated abelian-by-nilpotent group.

In [2] Baumslag, Myasnikov and Remeslennikov have developed algebraic geometry over groups. We recall here some definitions and results that we require. Let $G$ be a fixed group and $\bar{x}=\left(x_{1}, \ldots, x_{n}\right)$. We denote by $G[\bar{x}]$ the group $G * F(\bar{x})$ where $F(\bar{x})$ is the free group with basis $\left\{x_{1}, \ldots, x_{n}\right\}$.

For an element $s(\bar{x}) \in G[\bar{x}]$ and a tuple $\bar{g}=\left(g_{1}, \ldots, g_{n}\right) \in G^{n}$ we denote by $s(\bar{g})$ the element of $G$ obtained by replacing each $x_{i}$ by $g_{i}(1 \leq i \leq n)$. Let $S$ 
be a subset of $G[\bar{x}]$. Then the set

$$
V(S)=\left\{\bar{g} \in G^{n} \mid s(\bar{g})=1 \text { for all } s \in S\right\}
$$

is called the algebraic set over $G$ defined by $S$. For instance the centralizer of any element in $G$ is an algebraic set. Subsets of $G[\bar{x}]$ will be also seen as system of equations with parameters from $G$. A group $G$ is called equationally noetherian if for every $n \geq 1$ and every subset $S$ of $G[\bar{x}]$ there exists a finite subset $S_{0} \subseteq S$ such that $V(S)=V\left(S_{0}\right)$. A subset of $G^{n}$ is closed if it is the intersection of an arbitrary number of finite unions of algebraic sets. This defines a topology on $G^{n}$, called the Zariski topology. Then a group $G$ is equationally noetherian if and only if for each $n \geq 1$ the Zariski topology on $G^{n}$ is noetherian [2, Theorem D1].

Guba has shown that free groups are equationally noetherian using the fact that a free group is linear [8]. Using the same argument Baumslag, Myasnikov and Remeslennikov have proved that linear groups over a commutative, noetherian, unitary ring, e. g. a field are equationally noetherian $[2$, Theorem B1]. They have also shown that abelian groups are equationally noetherian. Bryant proved that finitely generated abelian-by-nilpotent groups are equationally noetherian [4]. Since the wreath product of a nontrivial finitely generated abelian group $G$ by a finitely generated nilpotent group $H$ is linear if and only if $H$ is virtually abelian [22], this provides examples of equationally noetherian groups that are not linear [2, p. 38]. Sela has shown that torsion-free hyperbolic groups are equationally noetherian [21, Theorem 1.22]. This answers a question in [2] and gives another proof of the fact that a free group is equationally noetherian. All of this shows that the class of equationally noetherian groups is very large.

We recall the definition of an $H$-limit group. Let $H$ and $G$ be groups. Let $\left(f_{i \in \mathbb{N}} \mid f_{i}: G \rightarrow H\right)$ be a sequence of morphisms from $G$ to $H$. We say that $f_{i \in \mathbb{N}}$ is convergent or stable if for every $g \in G$ one of the following sets is finite

$$
\left\{i \in \mathbb{N} \mid f_{i}(g)=1\right\},\left\{i \in \mathbb{N} \mid f_{i}(g) \neq 1\right\} .
$$

We let $\operatorname{ker}\left(f_{i}\right)=\left\{g \in G \mid\right.$ the set $\left\{i \in \mathbb{N} \mid f_{i}(g) \neq 1\right\}$ is finite $\}$. A group $K$ is said to be $H$-limit if there exist a group $G$ and a converging sequence $\left(f_{i \in \mathbb{N}} \mid f_{i}: G \rightarrow H\right)$ such that $K=G / \operatorname{ker}\left(f_{i}\right)$. In general limit groups are supposed finitely generated, but since some results do not depend on the finite generation property, we do not suppose that and we work in the general case. Thus, in our definition, the free group $F_{\aleph_{0}}$ of rank $\aleph_{0}$ is a limit group of free groups, though it is not finitely generated.

Limit groups are heavily connected to residual properties. Let $G$ be a group and $\mathcal{K}$ a class of groups. We say that $G$ is residually- $\mathcal{K}$ (or that $\mathcal{K}$ separates $G$ ) if for every $g \in G \backslash\{1\}$ there exist $K \in \mathcal{K}$ and a morphism $f: G \rightarrow K$ such that $f(g) \neq 1$. If $\mathcal{K}$ consists of the singleton $K$, then we say simply that $G$ is residually- $K$. We say that $G$ is fully residually- $\mathcal{K}$ (or that $\mathcal{K}$ discriminates $G$ ) if for every finite subset $X \subseteq G \backslash\{1\}$ there exist $K \in \mathcal{K}$ and a morphism 
$f: G \rightarrow K$ such that $1 \notin f(X)$. As before, if $\mathcal{K}$ consists of the singleton $K$, then we say simply that $G$ is fully residually- $K$.

We finish with some notions from Model Theory needed in the sequel. For more details the reader is referred to $[6,10,15]$.

The universal theory of a group $H$, denoted $\operatorname{Th}_{\forall}(H)$, is the set of all universal sentences true in $H$; i.e. sentences of the form

$$
\forall \bar{x}\left(\bigvee_{1 \leq i \leq m}\left(\bigwedge_{w \in P_{i}} w(\bar{x})=1 \wedge \bigwedge_{v \in N_{i}} v(\bar{x}) \neq 1\right)\right)
$$

(true in $H$ ), where $P_{i}, N_{i}$ are finite sets of words on the variables $\left\{x_{1}, \ldots, x_{n}\right\}$ and their inverses. An universal sentence with parameters from $H$ is defined analogously by allowing in the above words parameters from $H$.

The universal Horn theory of a group $H$, is the set of all sentences of the form

$$
\forall \bar{x}\left(\left(\bigwedge_{w \in P} w(\bar{x})=1\right) \Rightarrow v(\bar{x})=1\right)
$$

true in $H$, where $P$ is a finite set of words on the variables $\left\{x_{1}, \ldots, x_{n}\right\}$ and their inverses.

When $\phi$ is a sentence, we write $G \models \phi$ to mean that $\phi$ is true in $G$ and we say that $G$ is a model of $\phi . G \models \operatorname{Th}_{\forall}(H)$ means that $G$ satisfies every sentence $\phi \in \operatorname{Th}_{\forall}(H)$ and we say that $G$ is a model of $\operatorname{Th}_{\forall}(H)$ or that $G$ is a model of the universal theory of $H$.

An ultrafilter on a set $I$ is a finitely additive probability measure $\mu: \mathcal{P}(I) \rightarrow$ $\{0,1\}$. An ultrafilter $\mu$ is called nonprincipal if $\mu(X)=0$ for every finite subset $X \subseteq I$.

Given an ultrafilter $\mu$ on $I$ and a sequence of groups $\left(G_{i}\right)_{i \in I}$ we define an equivalence relation $\sim_{\mu}$ on $\prod_{i \in I} G_{i}$ by

$$
\hat{a}=\left(a_{i} \in G_{i}\right)_{i \in I} \sim_{\mu} \hat{b}=\left(b_{i} \in G_{i}\right)_{i \in I} \text { if and only if } \mu\left(\left\{i \in I \mid a_{i}=b_{i}\right\}\right)=1 .
$$

The set of equivalence classes $\left(\prod_{i \in I} G_{i}\right) / \sim_{\mu}$ is endowed with a structure of group by defining

$$
\hat{a} . \hat{b}=\hat{c} \text { if and only if } \mu\left(\left\{i \in I \mid a_{i} \cdot b_{i}=c_{i}\right\}\right)=1 .
$$

The group $\left(\prod_{i \in I} G_{i}\right) / \sim_{\mu}$ is called the ultraproduct of the family $\left(G_{i}\right)_{i \in I}$. When $G_{i}=G$ for all $i \in I,\left(\prod_{i \in I} G_{i}\right) / \sim_{\mu}$ is called an ultrapower and it is denoted by ${ }^{*} G$. If $\mu$ is nonprincipal, then ${ }^{*} G$ is called a nonprincipal ultrapower.

Define $\pi: G \rightarrow{ }^{*} G$ by $\pi(g)=\left(g_{i}=g \mid i \in I\right)$. Then $\pi$ is an embedding. Moreover, a theorem of $\operatorname{Los}$ [6, Theorem 4.1.9] claims that $G$ is an elementary subgroup of ${ }^{*} G$; that is, any sentence with parameters from $G$ which is true in $G$ is also true in ${ }^{*} G$. As consequence, we get the property, needed in the sequel, that if $G=\phi$, where $\phi$ is an universal sentence with parameters from $G$, then ${ }^{*} G \models \phi$. In particular ${ }^{*} G$ and $G$ have the same universal theory.

Another theorem in Model theory, due to Keisler ([6, Theorem 6.1.1] or [15, Exercice 4.5.37]), claims that when $I$ is countable, a nonprincipal ultrapower 
${ }^{*} G$ of $G$ is $\aleph_{1}$-saturated. In what follows, we need only a special case of that saturation property (compactness): if

$$
{ }^{*} G \models \forall \bar{x}\left(\bigvee_{i \in \mathbb{N}} \phi_{i}(\bar{x})\right),
$$

where each $\phi_{i}$ is of the form $\left(\bigwedge_{1 \leq j \leq p} w_{j}(\bar{x})=1 \wedge \bigwedge_{1 \leq j \leq q} v_{j}(\bar{x}) \neq 1\right)$, then there exists $n \in \mathbb{N}$ such that

$$
{ }^{*} G \models \forall \bar{x}\left(\bigvee_{0 \leq i \leq n} \phi_{i}(\bar{x})\right) .
$$

(The words $w_{j}(\bar{x}), v_{j}(\bar{x})$ are allowed to have parameters from ${ }^{*} G$.)

\section{Conventions.}

- In what follows, for a finitely generated group $G$ we write $G=\langle\bar{x} \mid P(\bar{x})\rangle$ to mean that $G$ is generated by $\bar{x}=\left(x_{1}, \ldots, x_{n}\right)$ and $P(\bar{x})$ is a presentation closed by deduction; that is, if $G=w(\bar{x})=1$ then $w \in P$. If $S(\bar{x})$ is a set of words we write $S(\bar{x})=1$ as abbreviation of the formula $\bigwedge_{w \in S} w(\bar{x})=1$.

- Let $G$ be an equationally noetherian group and let $S \subseteq G[\bar{x}]$. A subset $S_{0} \subseteq S$ is called a $G$-witness of $S$ if $S_{0}$ is finite and $V(S)=V\left(S_{0}\right)$. Notice that we have

$$
G \models \forall \bar{x}\left(S_{0}(\bar{x})=1 \Leftrightarrow S(\bar{x})=1\right) .
$$

- In all of the rest of this paper, all the ultrafilter considered will be on $\mathbb{N}$.

\section{First Properties}

We begin this section by the following theorem which regroups several relations between the different notions given in the introduction.

Theorem 2.1 Let $H$ be a group.

(1) A countable fully residually- $H$ group is an $H$-limit group.

(2) An $H$-limit group is embeddable in all nonprincipal ultrapowers of $H$; in particular it is a model of $\operatorname{Th}_{\forall}(H)$.

(3) Let $G$ be a finitely generated group. The following properties are equivalents:

(i) $G$ is a model of $\operatorname{Th}_{\forall}(H)$,

(ii) $G$ is embeddable in some nonprincipal ultrapower of $H$,

(iii) $G$ is embeddable in all nonprincipal ultrapowers of $H$.

(4) If $H$ is equationally noetherian then for every finitely generated group $G$ the following properties are equivalent:

(i) $G$ is a model of $\operatorname{Th}_{\forall}(H)$,

(ii) $G$ is fully residually- $H$,

(iii) $G$ is an $H$-limit group.

(5) If $H$ is equationally noetherian then for every finitely generated group $G$ the following properties are equivalent: 
(i) $G$ is a model of the universal Horn theory of $H$,

(ii) $G$ is residually- $H$.

The following Lemma is classical in Model Theory, but for completeness we provide a proof.

Lemma 2.2 Let $G=\langle\bar{x} \mid P(\bar{x})\rangle$ be a finitely generated group and $H$ a group. Let $N(\bar{x})$ be the set of all words $w(\bar{x})$ such that $G \models w(\bar{x}) \neq 1$. Suppose that for every finite subset $X \subseteq P(\bar{x}) \cup N(\bar{x})$, there exists a tuple $\bar{a}$ in $H$ such that $H=\bigwedge_{w \in X \cap P} w(\bar{a})=1 \wedge \bigwedge_{v \in X \cap N} v(\bar{a}) \neq 1$. Then $G$ is embeddable in all nonprincipal ultrapowers of $H$. In particular $G$ is a model of the universal theory of $H$.

\section{Proof}

Let $\mu$ be a nonprincipal ultrafilter on $\mathbb{N}$ and let ${ }^{*} H$ be the ultrapower of $H$ relatively to $\mu$.

Write $P(\bar{x}) \cup N(\bar{x})=\bigcup_{i \in \mathbb{N}} S_{i}$, where each $S_{i}$ is finite and $S_{i} \subseteq S_{i+1}$. For every $i \in \mathbb{N}$, pick $\bar{a}_{i} \in H$ such that $H \models \bigwedge_{w \in S_{i} \cap P} w\left(\bar{a}_{i}\right)=1 \bigwedge_{v \in S_{i} \cap N} v\left(\bar{a}_{i}\right) \neq 1$.

We claim that the map $f: G \rightarrow{ }^{*} H$ defined by $f(\bar{x})=\left(\bar{a}_{i} \mid i \in \mathbb{N}\right)$ extends to an embedding.

If $w(\bar{x}) \in P$, then there exists $n \in \mathbb{N}$ such that $w \in S_{n}$ and thus $H \models$ $w\left(\bar{a}_{i}\right)=1$ for every $i \geq n$. Therefore $\mu\left(\left\{i \in \mathbb{N}|H|=w\left(\bar{a}_{i}\right)=1\right\}\right)=1$ and thus ${ }^{*} H \models w\left(\left(\bar{a}_{i} \mid i \in \mathbb{N}\right)\right)=1$.

Similarly, if $v(\bar{x}) \in N$, then there exists $n \in \mathbb{N}$ such that $v \in S_{n}$ and thus $H \models v\left(\bar{a}_{i}\right) \neq 1$ for every $i \geq n$. As before, $\mu\left(\left\{i \in \mathbb{N}|H|=v\left(\bar{a}_{i}\right) \neq 1\right\}\right)=1$ and thus ${ }^{*} H \models v\left(\left(\bar{a}_{i} \mid i \in \mathbb{N}\right)\right) \neq 1$. This ends the proof of our claim.

Now, since universal sentences are conserved by taking subgroups, $G$ is a model of the universal theory of ${ }^{*} H$. As $H$ and ${ }^{*} H$ have the same universal theory, $G$ is a model of the universal theory of $H$.

Proof of Theorem 2.1.

(1). Let $G$ be a countable fully residually- $H$ group and write $G \backslash\{1\}=\bigcup_{i \in \mathbb{N}} S_{i}$ where each $S_{i}$ is finite and $S_{i} \subseteq S_{i+1}$. Then there exists a convergent sequence $\left(f_{i \in \mathbb{N}} \mid f_{i}: G \rightarrow H\right)$ such that $1 \notin f_{i}\left(S_{i}\right)$. Clearly $\underset{\operatorname{ker}}{\longrightarrow}\left(f_{i}\right)=1$ and thus $G$ is an $H$-limit group.

(2). Let $K$ be an $H$-limit group and $G$ be a group for which there exists a convergent sequence $\left(f_{i \in \mathbb{N}} \mid f_{i}: G \rightarrow H\right)$ such that $K=G / \underset{\operatorname{ker}}{\longrightarrow}\left(f_{i}\right)$. Let $\mu$ be a nonprincipal ultrafilter on $\mathbb{N}$, and let ${ }^{*} G$ (resp. ${ }^{*} H$ ) be the ultrapower of $G$ (resp. $H$ ) relatively to $\mu$. Let $\pi: G \rightarrow{ }^{*} G$ be the natural embedding defined by $g \rightarrow \hat{g}=\left(g_{i}=g \mid i \in \mathbb{N}\right)$. The map $f:{ }^{*} G \rightarrow{ }^{*} H$ defined by $f\left(\left(g_{i} \mid i \in \mathbb{N}\right)\right)=\left(f_{i}\left(g_{i}\right) \mid i \in \mathbb{N}\right)$ is a morphism and satisfies

$$
f\left(\left(g_{i} \mid i \in \mathbb{N}\right)\right)=1 \Leftrightarrow \mu\left(\left\{i \in \mathbb{N} \mid f_{i}\left(g_{i}\right)=1\right\}\right)=1 .
$$

Therefore, the morphism $\varphi=f \circ \pi: G \rightarrow{ }^{*} H$ satisfies

$$
\varphi(g)=1 \Leftrightarrow \mu\left(\left\{i \in \mathbb{N} \mid f_{i}(g)=1\right\}\right)=1 .
$$


Since $\left(f_{i \in \mathbb{N}} \mid f_{i}: G \rightarrow H\right)$ is a convergent sequence and $\mu$ is nonprincipal we get

$$
\varphi(g)=1 \Leftrightarrow\left\{i \in \mathbb{N} \mid f_{i}(g) \neq 1\right\} \text { is finite. }
$$

Hence $\operatorname{ker}(\varphi)=\underset{\operatorname{ker}}{\longrightarrow}\left(f_{i}\right)$. Thus $K=G / \operatorname{ker}(\varphi)$ and $K$ is embeddable in ${ }^{*} H$. In particular $K \models \operatorname{Th}_{\forall}(H)$.

$(3)(i i i) \Rightarrow(i i)$ is obvious, $(3)(i i) \Rightarrow(i)$ is also obvious as universal sentences are preserved by subgroups and $\operatorname{Th}_{\forall}(H)=\mathrm{Th}_{\forall}\left({ }^{*} H\right)$. The implication $(3)(i) \Rightarrow(i i)$ is a consequence of $(3)(i) \Rightarrow($ iii $)$ and of the existence of nonprincipal ultrafilters on $\mathbb{N}$; so it remains to show $(3)(i) \Rightarrow($ iii $)$.

Write $G=\langle\bar{x} \mid P(\bar{x})\rangle$ and let $N(\bar{x})$ be the set of words $v(\bar{x})$ such that $G \models v(\bar{x}) \neq 1$. Since $G$ is a model of the universal theory of $H$, for every finite subset $X \subseteq P(\bar{x}) \cup N(\bar{x})$, there exists a tuple $\bar{a}$ in $H$ such that $H \models \bigwedge_{w \in X \cap P} w(\bar{a})=1 \wedge \bigwedge_{v \in X \cap N} v(\bar{a}) \neq 1$. Therefore, by Lemma 2.2, $G$ is embeddable in all nonprincipal ultrapowers of $H$.

(4) $(i i) \Rightarrow(i i i)$ is a consequence of (1) and $(4)(i i i) \Rightarrow(i)$ is a consequence of (2), it remains to show $(4)(i) \Rightarrow(i i)$. Write $G=\langle\bar{x} \mid P(\bar{x})\rangle$ and let $P_{0}(\bar{x})$ be an $H$-witness of $P(\bar{x})$. Since $G \models \operatorname{Th}_{\forall}(H)$, for any words $v_{1}(\bar{x}), \ldots, v_{n}(\bar{x})$ such that $G \models \bigwedge_{1 \leq i \leq n} v_{i}(\bar{x}) \neq 1, H$ is a model of

$$
\exists \bar{x}\left(P_{0}(\bar{x})=1 \wedge \bigwedge_{1 \leq i \leq n} v_{i}(\bar{x}) \neq 1\right) .
$$

Therefore there exists $\bar{a} \in H$ satisfying $P(\bar{a})=1 \wedge \bigwedge_{1 \leq i<n} v_{i}(\bar{a}) \neq 1$. Since $H \models \forall \bar{x}\left(P_{0}(\bar{x})=1 \Leftrightarrow P(\bar{x})=1\right)$ there exists a morphism $\bar{f}: G \rightarrow H$ such that $f\left(v_{i}(\bar{x})\right)=v_{i}(\bar{a}) \neq 1,1 \leq i \leq n$. Thus $G$ is fully residually- $H$.

$(5)(i) \Rightarrow(i i)$. Write $G=\langle\bar{x} \mid P(\bar{x})\rangle$ and let $P_{0}(\bar{x})$ be an $H$-witness of $P(\bar{x})$. Since $G$ is a model of the universal Horn theory of $H$, for any word $v(\bar{x})$ such that $G \models v(\bar{x}) \neq 1, H$ is a model of $\exists \bar{x}\left(P_{0}(\bar{x})=1 \wedge v(\bar{x}) \neq 1\right)$. As before, there exists $\bar{a} \in H$ satisfying $P(\bar{a})=1 \wedge v(\bar{a}) \neq 1$. Thus there exists a morphism $f: G \rightarrow H$ such that $f(v(\bar{x})) \neq 1$. Thus $G$ is residually- $H$.

$(5)(i i) \Rightarrow(i)$. Clearly if $G$ is residually- $H$, then $G$ is embeddable in some product $\prod_{i \in I} H$ and thus $G$ is a model of the universal Horn theory of $H$.

\section{Remarks 2.3}

(1). The implications (4)(ii) $\Rightarrow(i)$ and $(5)(i i) \Rightarrow(i)$ do not depend on the equationally noetherian nature of $H$ neither on the finite generation property of $G$; that is $(4)(i i) \Rightarrow(i)$ and $(5)(i i) \Rightarrow(i)$ are still true for any group $H$ and any group $G$. The implication $(5)(i i) \Rightarrow(i)$ is very clear. Let us show that $(4)(i i) \Rightarrow(i)$ is true for any group $H$ and any group $G$. Suppose that $G$ is fully residually- $H$. Suppose

$$
H=\forall \bar{x}\left(\bigvee_{1 \leq i \leq m}\left(\bigwedge_{w \in P_{i}} w(\bar{x})=1 \wedge \bigwedge_{v \in N_{i}} v(\bar{x}) \neq 1\right)\right)
$$


where $P_{i}, N_{i}$ are finite sets of words, and suppose towards a contradiction

$$
G \models\left(\bigwedge_{1 \leq i \leq m}\left(\bigvee_{w \in P_{i}} w(\bar{a}) \neq 1 \vee \bigvee_{v \in N_{i}} v(\bar{a})=1\right)\right) \text {, for some tuple } \bar{a} \text { in } G
$$

Let

$$
X=\left\{w(\bar{a})\left|w(\bar{x}) \in P_{1} \cup \cdots \cup P_{m}, G\right|=w(\bar{a}) \neq 1\right\} .
$$

Since $G$ is fully residually- $H$, there exists a morphism $f: G \rightarrow H$ such that $1 \notin f(X)$. Then

$$
H \models\left(\bigwedge_{1 \leq i \leq m}\left(\bigvee_{w \in P_{i}} w(f(\bar{a})) \neq 1 \vee \bigvee_{v \in N_{i}} v(f(\bar{a}))=1\right)\right),
$$

contradicting $(*)$.

(2). Note that the equivalences (4)(i) - (iii) are not true if $G$ is not finitely generated: $\mathbb{Q}$ is a model of the universal theory of $\mathbb{Z}$ but $\mathbb{Q}$ is not residually- $\mathbb{Z}$. Furtheremore, $\mathbb{Q}$ is embeddable in all nonprincipal ultrapowers of $\mathbb{Z}$.

Corollary 2.4 Let $H$ be an equationally noetherian group. Then a group $G$ is a model of $T h_{\forall}(H)$ if and only if $G$ is locally fully residually- $H$.

Definition 2.5 A group $G$ is said $H$-pseudo-limit if $G$ is a model of the universal Horn theory of $H$.

In order to prove the next theorem we need the following definitions from [2]. Let $Y \subseteq G^{n}$. We define

$$
I(Y)=\{w(\bar{x}) \in G[\bar{x}] \mid w(\bar{y})=1 \text { for all } \bar{y} \in Y\} .
$$

Notice that when $S \subseteq G[\bar{x}]$, then

$$
w(\bar{x}) \in I(V(S)) \Leftrightarrow G \models \forall \bar{x}(S(\bar{x})=1 \Rightarrow w(\bar{x})=1) .
$$

For $S \subseteq G[\bar{x}]$ the coordinate group of $S$, denoted $G_{S}$, is $G[\bar{x}] / I(V(S))$. The proof of the following Lemma can certainly be extracted from [16] but for completeness we provide a proof.

Lemma 2.6 For any group $H$ and any $S \subseteq H[\bar{x}], H_{S}$ is residually- $H$. In particuliar $H_{S}$ is an $H$-pseudo-limit group.

\section{Proof}

Let $v(\bar{x})$ be a word such that $H_{S} \models v(\bar{x}) \neq 1$. Clearly we have $v(\bar{x}) \notin$ $I(V(S))$ and thus

$$
H \models \exists \bar{x}(S(\bar{x})=1 \wedge v(\bar{x}) \neq 1) .
$$

Thus there exists a morphism $f: H_{S} \rightarrow H$ which fixes every element of $H$ and $f(v(\bar{x})) \neq 1$. Therefore $H_{S}$ is residually- $H$ as desired.

By Remarks $2.3(1), H_{S}$ is an $H$-pseudo-limit group. 
Theorem 2.7 If $H$ is equationally noetherian then any sequence of epimorphisms of finitely generated $H$-pseudo-limit groups

$$
G_{1} \rightarrow_{\phi_{1,2}} G_{2} \rightarrow_{\phi_{2,3}} \cdots
$$

terminates after finitely many steps.

Conversely, if $H$ is finitely generated and any sequence of epimorphisms of finitely generated resiudally- $H$ groups terminates after finitely many steps, then $H$ is equationally noetherian.

\section{Proof}

Suppose that $H$ is equationally noetherian and write $\bar{x}=\left(x_{1}, \ldots, x_{n}\right)$, $G_{i}=\left\langle\bar{x} \mid P_{i}(\bar{x})\right\rangle, \phi_{i, i+1}\left(x_{k}\right)=x_{k}$. Since $H$ is equationally noetherian there exists a finite subset $S(\bar{x}) \subseteq P(\bar{x})=\bigcup_{i} P_{i}(\bar{x})$ such that

$$
H \models \forall \bar{x}(S(\bar{x})=1 \Rightarrow w(\bar{x})=1)) \text { for every } w \in P .
$$

Let $m \in \mathbb{N}$ such that $S(\bar{x}) \subseteq P_{m}(\bar{x})$. We claim that for every $k \geq m$, the epimorphism $\phi_{k, k+1}: G_{k} \rightarrow G_{k+1}$ is an isomorphism. Let $v(\bar{x})$ be a word on $\bar{x}$ such that $G_{k+1} \models v(\bar{x})=1$. Then there exists a finite subset $A \subseteq P_{k+1}$ such that $\forall \bar{x}(A(\bar{x})=1 \Rightarrow v(\bar{x})=1)$ is true in any group. Since $k+1 \geq m$ we have

$$
H \models \forall \bar{x}(S(\bar{x})=1 \Rightarrow A(\bar{x})=1)),
$$

thus we get

$$
H \models \forall \bar{x}(S(\bar{x})=1 \Rightarrow v(\bar{x})=1)) .
$$

Since $G_{k}$ is a model of the universal Horn theory of $H$, we get $G_{k} \models v(\bar{x})=1$.

Now suppose that $H$ is finitely generated and that any sequence of epimorphisms of finitely generated residually- $H$ groups terminates after finitely many steps. Let $S(\bar{x})$ be a system of equations with parameters from $H$. Since $H$ is finitely generated $S(\bar{x})$ is countable. Write $S(\bar{x})=\bigcup_{i \in \mathbb{N}} S_{i}(\bar{x})$ where $S_{i}$ is finite and $S_{i} \subseteq S_{i+1}$. Let $P_{i}(\bar{x})$ be the set of words $w(\bar{x})$ with parameters from $H$ such that

$$
H \models \forall \bar{x}\left(S_{i}(\bar{x})=1 \Rightarrow w(\bar{x})=1\right),
$$

i.e. $P_{i}(\bar{x})=I\left(V\left(S_{i}\right)\right)$. Let $H_{i}=\left\langle H * F(\bar{x}) \mid P_{i}(\bar{x})\right\rangle=H_{S_{i}}$. By Lemma $2.6, H_{i}$ is residually- $H$. Now the sequence

$$
H_{1} \rightarrow H_{2} \rightarrow H_{3} \cdots
$$

is a sequence of epimorphisms of finitely generated residually- $H$ groups, and thus stabilizes. Therefore there exists $n$ such that for every $i, j \geq n, P_{i}(\bar{x})=P_{j}(\bar{x})$. Hence for every $i \geq n$ we have

$$
H \models \forall \bar{x}\left(S_{n}(\bar{x})=1 \Rightarrow S_{i}(\bar{x})=1\right) .
$$

Thus $S(\bar{x})$ is equivalent to a finite subsystem as desired.

By Theorem 2.1 (2) an $H$-limit group is a model of the universal theory of $H$ and thus it is $H$-pseudo-limit. Thus we get: 
Corollary 2.8 Let $H$ be an equationally noetherian group. Then any sequence of epimorphisms of finitely generated $H$-limit groups

$$
G_{1} \rightarrow G_{2} \rightarrow \cdots
$$

terminates after finitely many steps.

Corollary 2.9 Let $H$ be an equationally noetherian group. Then every finitely generated $H$-pseudo-limit group is Hopfian. In particular every $H$-limit group is Hopfian.

\section{Proof}

Let $G$ be a finitely generated $H$-pseudo-limit group and $\phi: G \rightarrow G$ be a surjective morphism. Then the sequence $G \rightarrow_{\phi} G \rightarrow_{\phi} G \rightarrow_{\phi} G \cdots$ terminates after finitely many steps. Thus $\phi$ is an isomorphism.

Corollary 2.10 Let $H$ be an equationally noetherian group. Then any finitely generated $H$-pseudo-limit group is equationally noetherian. In particular any finitely generated $\mathrm{H}$-limit group is equationally noetherian.

\section{Proof}

Let $G$ be finitely generated $H$-pseudo-limit group. Then any sequence of epimorphisms of finitely generated residually- $G$ groups is also a sequence of epimorphisms of finitely generated $H$-pseudo-limit groups; thus terminates after finitely many steps, by Theorem 2.7. Again by Theorem $2.7, G$ is equationally noetherian.

Theorem 2.11 Let $H$ be an equationally noetherian group. Then there exists at most countably many nonisomorphic finitely generated $H$-pseudo-limit groups. In particular there exists at most countably many nonisomorphic finitely generated $H$-limit groups.

\section{Proof}

Suppose towards a contradiction that the opposite is true. Then there exists $n \in \mathbb{N}$ such that there exists at least $\lambda$ nonisomorphic $n$-generated $H$-pseudolimit groups for some $\lambda>\aleph_{0}$, ( $n$-generated means generated by $n$ elements). (In fact we can assume that $\lambda=2^{\aleph_{0}}$, see [18]). Let $\left(G_{i}=\left\langle\bar{x} \mid P_{i}(\bar{x})\right\rangle \mid i \in \lambda>\aleph_{0}\right)$ be the list of nonisomorphic $n$-generated $H$-pseudo-limit groups. For every $i \in \lambda$ there exists a finite subset $S_{i} \subseteq P_{i}$ such that $H \models \forall \bar{x}\left(S_{i}(\bar{x})=1 \Leftrightarrow P_{i}(\bar{x})=1\right)$.

Since for every $i \in \lambda$ the set $S_{i}$ is finite, the set $\left\{S_{i} \mid i \in \lambda\right\}$ is countable. Therefore the map $f:\left\{P_{i} \mid i \in \lambda\right\} \rightarrow\left\{S_{i} \mid i \in \lambda\right\}$ defined by $P_{i} \mapsto S_{i}$ is not injective and thus there exist $i, j \in \lambda, i \neq j$ such that $S_{i}=S_{j}$.

Since $G_{i}, G_{j}$ are models of the universal Horn theory of $H$ we get $P_{i}=P_{j}$, a contradiction with the fact that $G_{i}$ and $G_{j}$ are not isomorphic. 


\section{$3 \quad$ Factor sets}

Let $H$ be a group equipped with the Zariski topology. Recall that a closed set is called irreducible if it is not the union of two proper, nonempty, closed subsets. In our context, if $S \subseteq H[\bar{x}]$ is a system of equations such that $V(S)$ is irreducible, then whenever $S_{1}, \ldots, S_{n} \subseteq H[\bar{x}]$ are systems of equations and if

$$
H \models \forall \bar{x}\left(S(\bar{x})=1 \Rightarrow S_{1}(\bar{x})=1 \vee \cdots \vee S_{n}(\bar{x})=1\right),
$$

then there exists $i$ such that $H=\forall \bar{x}\left(S(\bar{x})=1 \Rightarrow S_{i}(\bar{x})=1\right)$.

Now suppose that $H$ is equationally noetherian. As noticed in the introduction the Zariski topology on $H^{n}$ is noetherian. This implies that every closed subset of $H^{n}$ is a finite union of algebraic sets. Recall also that every closed set in a noetherian topological space is a finite union of irreducible closed subsets. Thus, in our context, for any system $S(\bar{x})$ of equations there exist systems of equations $S_{1}, \ldots, S_{n}$ such that $\left(V\left(S_{i}\right) \mid 1 \leq i \leq n\right)$ are irreducible and

$$
H \models \forall \bar{x}\left(S(\bar{x})=1 \Leftrightarrow S_{1}(\bar{x})=1 \vee \cdots \vee S_{n}(\bar{x})=1\right) .
$$

Some properties contained in the next Lemma, can certainly be extracted from $[2]$.

Lemma 3.1 Let $H$ be a group and $S \subseteq H[\bar{x}]$ such that $V(S)$ is irreducible. Then $H_{S}$ is fully residually-H. In particular, $H_{S}$ is a model of the universal theory of $H$, and if $H$ is countable then $H_{S}$ is an $H$-limit group.

\section{Proof} that

Let $v_{1}(\bar{x}), \ldots, v_{p}(\bar{x})$ be words such that $H_{S} \models \bigwedge_{1 \leq j \leq p} v_{j}(\bar{x}) \neq 1$. Suppose

$$
H \models \forall \bar{x}\left(S(\bar{x})=1 \Rightarrow \bigvee_{1 \leq j \leq p} v_{j}(\bar{x})=1\right) .
$$

Then, since $V(S)$ is irreducible, there exists $j$ such that

$$
H \models \forall \bar{x}\left(S(\bar{x})=1 \Rightarrow v_{j}(\bar{x})=1\right),
$$

contradicting the fact that $H_{S} \models v_{j}(\bar{x}) \neq 1$. Therefore

$$
H \models \exists \bar{x}\left(S(\bar{x})=1 \wedge \bigwedge_{1 \leq j \leq p} v_{j}(\bar{x}) \neq 1\right) .
$$

Consequently, there exists a morphism $f: H_{S} \rightarrow H$, which fixes every element of $H$, such that $f\left(v_{j}\right) \neq 1(1 \leq j \leq p)$. Thus $H_{S}$ is fully residually- $H$ as desired.

It follows, by Remarks $2.3(1)$, that $H_{S}$ is a model of the universal theory of $H$. Furtheremore, if $H$ is countable then $H_{S}$ is also countable and by Theorem 2.1 (1), $H_{S}$ is an $H$-limit group.

Factors sets are the first step in the construction of Makanin-Razborov diagrams. Let $H$ be a group, $K$ an $H$-limit group and $G$ a finitely generated 
group. A factor set of $G$ relatively to $K$ is a finite collection of proper quotients $\left\{f_{i}: G \rightarrow L_{i}\right\}$ of $H$-limit groups such that any morphism $f: G \rightarrow K$ factors through some $f_{i}$ after precomposition with some automorphism of $G$.

The following Theorem gives a weak version of factor sets.

Theorem 3.2 Let $H$ be an equationally noetherian group. Then for any finitely generated group $G$ there exists a finite collection of epimorphisms $\left\{f_{i}: G \rightarrow L_{i}\right\}$, where each $L_{i}$ is an $H$-limit group, such that for any $H$-limit group $L$ any morphism $f: G \rightarrow L$, factors through some $f_{i}$.

\section{Proof}

Put $G=\langle\bar{x} \mid P(\bar{x})\rangle$ and let $S(\bar{x})$ be an $H$-witness of $P$. Let $S_{1}, \ldots, S_{n}$ be systems of equations (maybe with parameters from $H$ ) such that each $S_{i}$ is finite, $\left(V\left(S_{i}\right) \mid 1 \leq i \leq n\right)$ are irreducible and

$$
H \models \forall \bar{x}\left(S(\bar{x})=1 \Leftrightarrow S_{1}(\bar{x})=1 \vee \cdots \vee S_{n}(\bar{x})=1\right) .
$$

Let $P_{i}$ be the set of words $w(\bar{x})$, without parameters from $H$, such that

$$
H \models \forall \bar{x}\left(S_{i}(\bar{x})=1 \Rightarrow w(\bar{x})=1\right) .
$$

Let $L_{i}=\left\langle\bar{x} \mid P_{i}(\bar{x})\right\rangle$. We claim that the sequence $L_{1}, \ldots, L_{n}$ satisfies the desired properties.

First we claim that $L_{i}$ is an $H$-limit group. Let us show that $L_{i}$ is the subgroup of $H_{S_{i}}$ generated by $\bar{x}$. Clearly the map $\phi: L_{i} \rightarrow H_{S_{i}}$ which sends $x_{i}$ to $x_{i}$ is a morphism. We claim that it is injective. Indeed if $v(\bar{x})$ is a word, without parameters from $H$, such that $H_{S_{i}} \models v(\bar{x})=1$ then $v(\bar{x}) \in I\left(V\left(S_{i}\right)\right)$ and thus $v(\bar{x}) \in P_{i}$. Therefore $L_{i}=v(\bar{x})=1$ and thus $\phi$ is injective.

By Lemma 3.1, $H_{S_{i}}$ is a model of $\operatorname{Th}_{\forall}(H)$ and thus $L_{i}$ is also a model of $\mathrm{Th}_{\forall}(H)$. As $L_{i}$ is finitely generated, by Theorem $2.1(4)(i) \Leftrightarrow(i i i), L_{i}$ is an $H$-limit group.

Since $L_{i}$ is an $H$-limit group and $L_{i} \models S(\bar{x})=1$, there is an epimorphism $f_{i}: G \rightarrow L_{i}$ such that $f_{i}\left(x_{k}\right)=x_{k}$.

Now let $L$ be an $H$-limit group and $h: G \rightarrow L$ be an epimorphism. Since $L \models$ $\mathrm{Th}_{\forall}(H)$, by Theorem 2.1 (3) $L$ is embeddable in some nonprincipal ultrapower ${ }^{*} H$ of $H$. Without loss of generality we may assume that $L \subseteq{ }^{*} H$. Write $L=\langle\bar{y} \mid Q(\bar{y})\rangle$ such that $h\left(x_{i}\right)=y_{i}$. Then $L \models P(\bar{y})=1$, and thus $L \models S(\bar{y})=1$. Since every universal sentence with parameters from $H$ is true in ${ }^{*} H$ (see the introduction) we get

$$
{ }^{*} H \models \forall \bar{x}\left(S(\bar{x})=1 \Leftrightarrow S_{1}(\bar{x})=1 \vee \cdots \vee S_{n}(\bar{x})=1\right) .
$$

Therefore there exists $j$ such that ${ }^{*} H \models S_{j}(\bar{y})=1$. Again, since every universal sentence with parameters from $H$ is true in ${ }^{*} H$, we get ${ }^{*} H \models P_{j}(\bar{y})=1$ and thus $L \models P_{j}(\bar{y})=1$. Hence there exists a morphism $\varphi: L_{j} \rightarrow L$ which sends $x_{i}$ to $y_{i}$. Now clearly $h=\varphi \circ f_{i}$.

Note that the proof of the above Theorem is slightly different of those presented in [7] for free groups, as here we do not use the fact that some ultrapower of $H$ is equationally noetherian. 
Corollary 3.3 Let $H$ be an equationally noetherian group. Then a finitely generated group $G$ is residually- $H$ if and only if there exist $H$-limit groups $L_{1}, \ldots, L_{n}$ such that $G$ is embeddable in $L_{1} \times \cdots \times L_{n}$.

\section{Proof}

Clearly if $G$ is embeddable in $L_{1} \times \cdots \times L_{n}$ for some $H$-limit groups $L_{1}, \ldots, L_{n}$ then $G$ is a model of the universal Horn theory of $H$ and thus it is residually- $H$ by Theorem 2.1(5).

Suppose that $G$ is residually- $H$. By Theorem 3.2, there exists a finite collection of epimorphisms $\left\{f_{i}: G \rightarrow L_{i}\right\}$, where each $L_{i}$ is an $H$-limit group, such that for any $H$-limit group $L$ any morphism $h: G \rightarrow L$, factors through some $f_{i}$. We claim that $G$ is embeddable in $K=L_{1} \times \cdots \times L_{m}$.

Write $G=\left\langle x_{1}, \ldots, x_{n} \mid P(\bar{x})\right\rangle$ and $L_{i}=\left\langle y_{i, 1}, \ldots, y_{i, n} \mid P_{i}\left(\bar{y}_{i}\right)\right\rangle$ such that $f_{i}\left(x_{j}\right)=y_{i, j}$. Then the map $f: G \rightarrow L_{1} \times \cdots \times L_{m}$ defined by

$$
f\left(x_{j}\right)=\hat{y}_{j}=\left(y_{1, j}, \ldots, y_{m, j}\right)
$$

is a morphism. It remains to show that $f$ is an embedding. Let $w\left(\hat{y}_{1}, \ldots, \hat{y}_{n}\right)$ be a word on the variables $\left\{\hat{y}_{1}, \ldots \hat{y}_{n}\right\}$ such that $w\left(\hat{y}_{1}, \ldots, \hat{y}_{n}\right)=1$ in $K$. Suppose towards a contradiction that $G \models w\left(x_{1}, \ldots, x_{n}\right) \neq 1$. Since $G$ is residually$H$ there exists a morphism $\phi: G \rightarrow H$ such that $\phi\left(w\left(x_{1}, \ldots, x_{n}\right)\right) \neq 1$. Now there exist $f_{i}$ and a morphism $h: L_{i} \rightarrow H$ such that $\phi=h \circ f_{i}$ and thus $h\left(w\left(y_{i, 1}, \ldots, y_{i, n}\right)\right) \neq 1$. Therefore $L_{i} \models w\left(y_{i, 1}, \ldots, y_{i, n}\right) \neq 1$ and thus $K=w\left(\hat{y}_{1}, \ldots, \hat{y}_{n}\right) \neq 1$, contradiction. Hence $f$ is an embedding as claimed.

A natural question arise from Theorem 3.2: when can we say that the groups $L_{i}$ are a proper quotients of $G$ ? Clearly if $G$ is not $H$-limit, then the groups $L_{i}$ are proper quotients. When $G$ is an $H$-limit group, to answer the question, we need the following notion.

Definition 3.4 A finitely generated $H$-limit group $G$ is said $H$-determined if there exists a finite subset $X \subseteq G \backslash\{1\}$ such that for any morphism $f: G \rightarrow L$, where $L$ is an $H$-limit group, if $1 \notin f(X)$ then $f$ is an embedding. We denote by $\mathcal{D}_{H}$ the class of $H$-determined groups.

Notice that every $H$-determined group is a subgroup of $H$. In particular if $H$ is free then any $H$-determined group is also free. For instance $\mathbb{Z}, F_{2}$ are $F_{2}$-determined but $\mathbb{Z}^{2}$ is not. Thus, in some sense, $H$-determined groups play the same role in general case, as free groups in the special case of limit groups of free groups. Notions related to determined groups are investigated in [3] and [17].

A primitive-quantifier-free formula is a formula $\vartheta(\bar{x})$ of the form

$$
\left.\left(\bigwedge_{w \in P} w(\bar{x})=1 \wedge \bigwedge_{v \in N} v(\bar{x}) \neq 1\right)\right)
$$

where $P, N$ are finite sets of words on the variables $\bar{x}=\left\{x_{1}, \ldots, x_{n}\right\}$ and their inverses. We begin with the following proposition. 
Proposition 3.5 [17, Proposition 2.3.1] Let $H$ be a group such that $T h_{\forall}(H)$ has at most countably many nonisomorphic finitely generated models. Then for any primitive-quantifier-free formula $\vartheta(\bar{x})$ such that $H=\exists \bar{x}(\vartheta(\bar{x}))$, there exists a primitive-quantifier-free formula $\xi(\bar{x})$ such that $H \models \exists \bar{x}(\vartheta(\bar{x}) \wedge \xi(\bar{x}))$ and for any word $w(\bar{x})$ on the variables $\bar{x}=\left\{x_{1}, \ldots, x_{n}\right\}$ and their inverses one has

$$
H \models \forall \bar{x}(\vartheta(\bar{x}) \wedge \xi(\bar{x}) \Rightarrow w(\bar{x})=1) \text { or } H \models \forall \bar{x}(\vartheta(\bar{x}) \wedge \xi(\bar{x}) \Rightarrow w(\bar{x}) \neq 1) .
$$

\section{Proof}

Let $\vartheta(\bar{x})$ be a primitive-quantifier-free formula such that $H \models \exists \bar{x}(\vartheta(\bar{x}))$ and suppose towards a contradiction that $\vartheta(\bar{x})$ does not satisfies the conclusions of the proposition. We are going to construct a tree. By hypothesis there exists a word $\alpha_{1}(\bar{x})$ such that $H \models \exists \bar{x}\left(\vartheta(\bar{x}) \wedge \alpha_{1}(\bar{x})=1\right)$ and $H \models \exists \bar{x}\left(\vartheta(\bar{x}) \wedge \alpha_{1}(\bar{x}) \neq 1\right)$ (to simplify notation we omit $\bar{x}$ ). We can do the same thing with $\vartheta \wedge \alpha_{1}=1$ and $\vartheta \wedge \alpha_{1} \neq 1$.

Thus we have:

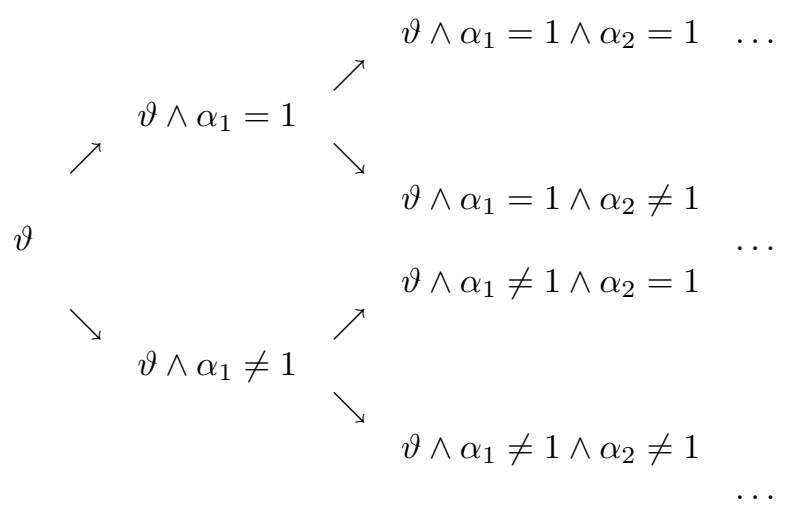

Now every branch $B$ in the above tree defines a finitely generated group $G_{B}$, by taking $G_{B}$ the group generated by $\bar{x}$ and with presentation the set of all words equal to 1 which appear in the branch $B$. Then $G_{B}$ satisfies any inequality which occurs in $B$. Since any finite segment in any branch $B$ is consistent in $H$, by Lemma $2.2, G_{B}$ is a model of the universal theory of $H$. Since there exists $2^{\aleph_{0}}$ branch, we get $2^{\aleph_{0}}$ nonisomorphic finitely generated models of $\operatorname{Th}_{\forall}(H)$. Contradiction with the property satisfied by $H$.

Theorem 3.6 Let $H$ be an equationally noetherian group and $G$ a nontrivial finitely generated $H$-limit group. Then for any finite subset $X \subseteq G \backslash\{1\}$ there exists an epimorphism $f: G \rightarrow L$ where $L$ is an $H$-determined group such that $1 \notin f(X)$.

\section{Proof}

Write $G=\langle\bar{x} \mid P(\bar{x})\rangle$ and let $S(\bar{x})$ be an $H$-witness of $P$. Let $X \subseteq G \backslash\{1\}$ be finite and let $v_{1}(\bar{x}), \ldots, v_{n}(\bar{x})$ be words representing the elements of $X$. 
Let $\vartheta(\bar{x})=\left(S(\bar{x})=1 \wedge \bigwedge_{1<i<n} v_{i}(\bar{x}) \neq 1\right)$. By Theorem 2.11, there exists countably many nonisomorphic $H$-limit groups and thus by Proposition 3.5 there exists a primitive-quantifier-free formula $\xi(\bar{x})$ such that $H=\exists \bar{x}(\vartheta(\bar{x}) \wedge \xi(\bar{x}))$ and for any word $w(\bar{x})$ on the variables $\bar{x}=\left\{x_{1}, \ldots, x_{n}\right\}$ and their inverses, one has

$$
H \models \forall \bar{x}(\vartheta(\bar{x}) \wedge \xi(\bar{x}) \Rightarrow w(\bar{x})=1) \text { or } H \models \forall \bar{x}(\vartheta(\bar{x}) \wedge \xi(\bar{x}) \Rightarrow w(\bar{x}) \neq 1) .
$$

Let $\left.\xi(\bar{x})=\left(\bigwedge_{w \in Q} w(\bar{x})=1 \wedge \bigwedge_{u \in N} u(\bar{x}) \neq 1\right)\right)$, where $Q, N$ are finite sets of words. Since $H \models \exists \bar{x}(\vartheta(\bar{x}) \wedge \xi(\bar{x}))$ there exists $\bar{a} \in H^{n}$ such that

$$
\left.H \models\left(S(\bar{a})=1 \wedge \bigwedge_{w \in Q} w(\bar{a})=1 \wedge \bigwedge_{1 \leq i \leq n} v_{i}(\bar{a}) \neq 1 \wedge \bigwedge_{u \in N} u(\bar{a}) \neq 1\right)\right) .
$$

Let $L=\langle\bar{a}\rangle$. Then $L$ is an $H$-limit group as it is a subgroup of $H$ and since $L \models S(\bar{a})=1$ there is an epimorphism $f: G \rightarrow L$ which sends $x_{i}$ to $a_{i}$.

We claim that $L$ is an $H$-determined group. Let $C$ be the set of elements of $L$ which are represented by the words $v_{1}(\bar{a}), \ldots, v_{n}(\bar{a})$ and $u(\bar{a}), u \in N$. Then for any morphism $h: L \rightarrow K$ where $K$ is an $H$-limit group if $1 \notin h(C)$ then

$$
K \models \vartheta(h(\bar{a})) \wedge \xi(h(\bar{a})),
$$

and thus, since $K$ is $H$-limit, by (1) we get

$$
L \models w(\bar{a})=1 \Leftrightarrow K \models w(h(\bar{a}))=1 .
$$

Therefore $h$ is an embedding. Thus $L$ is $H$-determined.

Theorem 3.7 Let $H$ be an equationally noetherian group. Then a finitely generated group $G$ is $H$-limit if and only if $G$ is fully residually- $\mathcal{D}_{H}$. Furtheremore, a finitely generated $H$-limit group $G$ has the same universal theory as $H$ if and only if every $H$-determined group is embeddable in $G$.

\section{Proof}

Let $G$ be a finitely generated $H$-limit group. Then, by Theorem 3.6, $G$ is fully residually- $\mathcal{D}_{H}$.

Now if $G$ is fully residually- $\mathcal{D}_{H}$, then clearly it is fully residually- $H$ as any $H$-determined group is a subgroup of $H$.

Let $G$ be a finitely generated group having the same universal theory as $H$. Let $L=\langle\bar{x} \mid P(\bar{x})\rangle$ be an $H$-determined group and let $X \subseteq G \backslash\{1\}$, given by words $v_{1}(\bar{x}), \cdots, v_{n}(\bar{x})$, such that for any morphism $f: G \rightarrow L$, where $L$ is an $H$-limit group, if $1 \notin f(X)$ then $f$ is an embedding. Let $S(\bar{x})$ be $H$-witness of $P$. Then

$$
H \models \exists \bar{x}\left(S(\bar{x})=1 \wedge \bigwedge_{1 \leq i \leq n} v_{i}(\bar{x}) \neq 1\right) .
$$

Since $\operatorname{Th}_{\forall}(G)=\operatorname{Th}_{\forall}(H), G$ satisfies the sentence which appears in (1). Therefore, as $L$ is $H$-determined and $G$ is $H$-limit, $L$ is embeddable in $G$. 
Now suppose that every $H$-determined group is embeddable in $G$ and let us show that $H$ is a model of the universal theory of $G$. Since $G$ is $H$-limit, by Corollary 2.10, $G$ is equationally noetherian. Therefore, by Corollary 2.4, it remains to show that every finitely generated subgroup of $H$ is $G$-limit. By the above result every finitely generated subgroup of $H$ is fully residually- $\mathcal{D}_{H}$. Therefore, every finitely generated subgroup of $H$ is fully residually- $G$ and thus it is $G$-limit.

Corollary 3.8 If $H$ is equationally noetherian then $T h_{\forall}(H)=\bigcap_{K \in \mathcal{D}_{H}} T h_{\forall}(K)$.

Corollary 3.9 If $H$ is equationally noetherian and if every $H$-determined group is residually-finite then every finitely generated $H$-limit group is residually-finite.

This justifies the following problem.

Problem. What are the $H$-determined groups for $H$ an hyperbolic group ?

Definition 3.10 A strong factor set for a group $G$ is a finite collection of proper quotients $\left\{f_{i}: G \rightarrow G_{i}\right\}$ of H-limit groups such that for any H-limit group $L$ and any morphism $f: G \rightarrow L$ either $f$ is an embedding or $f$ factors through some $f_{i}$.

Theorem 3.11 Let $H$ an equationally noetherian group. A nontrivial finitely generated $H$-limit group $G$ is $H$-determined if and only if $G$ has a strong factor set.

\section{Proof}

Let $G$ be a nontrivial finitely generated $H$-limit group which is $H$-determined and let us prove that $G$ has a strong factor set. If every proper quotient of $G$ which is $H$-limit is trivial then we have the desired conclusion. So suppose that $G$ has a nontrivial proper quotient which is $H$-limit. Write $G=\langle\bar{x} \mid P(\bar{x})\rangle$. By Theorem 2.11, $G$ has at most countably many nonisomorphic $H$-limit quotients. Let $\left(G_{i}=\left\langle\bar{x} \mid P_{i}(\bar{x})\right\rangle \mid i \in \mathbb{N}\right)$ be the list of all nontrivial proper quotients of $G$ which are $H$-limit. Since $H$ is equationally noetherian there exist finite subsets $S(\bar{x}) \subseteq P(\bar{x}), S_{i}(\bar{x}) \subseteq P_{i}(\bar{x})$ such that

$$
\begin{gathered}
H \models \forall \bar{x}(S(\bar{x})=1 \Leftrightarrow P(\bar{x})=1) . \\
H \models \forall \bar{x}\left(S_{i}(\bar{x})=1 \Leftrightarrow P_{i}(\bar{x})=1\right) .
\end{gathered}
$$

Since $G$ is $H$-determined there exists a finite number $v_{1}(\bar{x}), \ldots, v_{n}(\bar{x})$ of words such that if $K$ is an $H$-limit group containing $\bar{a}$ which satisfies $S(\bar{a})=$ $1 \wedge \bigwedge_{1<i<n} v_{i}(\bar{a}) \neq 1$, then the morphism $f: G \rightarrow K$ defined by $f\left(x_{i}\right)=a_{i}$ is an embeding. 
Let ${ }^{*} H$ be a nonprincipal ultrapower of $H$. Then every $H$-limit group is embeddable in ${ }^{*} H$. Now if $\bar{b} \in{ }^{*} H$ is a tuple such that ${ }^{*} H \models S(\bar{b})=1$, then

$$
{ }^{*} H \models \bigwedge_{1 \leq i \leq n} v_{i}(\bar{b}) \neq 1 \vee \bigvee_{j \in \mathbb{N}} S_{j}(\bar{b})=1
$$

Since ${ }^{*} H$ is $\aleph_{1}$-saturated, there is $m \in \mathbb{N}$ such that for every $\bar{b} \in{ }^{*} H$ satisfying ${ }^{*} H \models S(\bar{b})=1$ we get

$$
{ }^{*} H \models \bigwedge_{1 \leq i \leq n} v_{i}(\bar{b}) \neq 1 \vee \bigvee_{1 \leq j \leq m} S_{j}(\bar{b})=1 .
$$

Therefore

$$
H \models \forall \bar{x}\left(S(\bar{x})=1 \Rightarrow \bigwedge_{1 \leq i \leq n} v_{i}(\bar{x}) \neq 1 \vee \bigvee_{1 \leq j \leq m} S_{j}(\bar{x})=1\right)
$$

We claim that $\left\{G_{1}, \ldots, G_{m}\right\}$ is a strong factor set where $f_{i}$ is defined by the obvious manner. Let $f: G \rightarrow L$ be a morphism, where $L$ is an $H$-limit group. Then there exists $\bar{a} \in L$ such that $L \models S(\bar{a})=1$ and $f\left(x_{i}\right)=a_{i}$. Since $L$ is an $H$-limit group it satisfies the sentence appearing in (1) and thus $\langle\bar{a}\rangle$ is either isomorphic to $G$ or $\langle\bar{a}\rangle \models \bigvee_{1 \leq j \leq m} S_{j}(\bar{a})=1$. Thus if $f$ is not an embedding, there exists $j$ and a morphism $h: G_{j} \rightarrow\langle\bar{a}\rangle$ and we see that $f=f_{i} \circ h$. Thus $\left\{G_{1}, \ldots, G_{m}\right\}$ is a strong factor set as claimed.

Suppose now that $G$ has a strong factor set $\left\{f_{i}: G \rightarrow G_{i} \mid 1 \leq i \leq m\right\}$ and let us show that $G$ is $H$-determined.

Write $\left(G_{i}=\left\langle\bar{x} \mid P_{i}(\bar{x})\right\rangle \mid 1 \leq i \leq m\right)$ with $f\left(x_{i}\right)=x_{i}$. Since every $G_{i}$ is a proper quotient, there exists words $v_{1}(\bar{x}), \ldots, v_{m}(\bar{x})$ such that

$$
G_{i} \models v_{i}(\bar{x})=1, G \models v_{i}(\bar{x}) \neq 1 .
$$

Therefore if $f: G \rightarrow L$ where $L$ is an $H$-limit group such that $\bigwedge_{1 \leq i \leq m} f\left(v_{i}\right) \neq 1$ then clearly $f$ is an embedding.

\section{References}

[1] B. Baumslag. Residually free groups. Proc. London Math. Soc. (3) 17 (1967), 402-418.

[2] G. Baumslag, A. Myasnikov, V.N. Remeslennikov. Algebraic geometry over groups I: Algebraic sets and ideal theory. J. Algebra, 219 (1999), 16-79.

[3] O. Belegradek. Finitely determined members of varieties of groups and rings. J. Algebra, 228 (2000), 586-602.

[4] R. Bryant. The verbal topology of a group. J. Algebra 48 (1977), 340-346. 
[5] C. Champetier and V. Guirardel. Limit groups as limits of free groups: compactifyijng the set of free groups. Israel Journal of Mathematics, 146 (2005).

[6] C. C. Chang and H. J. Keissler. Model Theory. Studies in Logic and the foundations of mathematics, Volume 73, Elsevier Science, 1990.

[7] Z. Chatzidakis. Limit groups, viewed by a logician. Preprint available at http://www.logique.jussieu.fr/www.zoe

[8] V. Guba. Equivalence of infinite systems of equations in free groups and semigroups to finite subsystems. Mat. Zametki 40, NO. 3 (1986), 321-324.

[9] V. Guirardel. Limit groups and groups acting freely on $R^{n}$-trees. Geom. Topol. 8 (2004), 1427-1470.

[10] W. Hodges. Model theory, volume 42 of Encyclopedia of Mathematics and its Applications. Cambridge University Press, Cambridge, 1993.

[11] I. Kapovich. Subgroup properties of fully residually free groups. Trans. Amer. Math. Soc. 354 (2002), 335-362.

[12] O. Kharlampovich and A. Myasnikov. Irreducible affine varieties over a free group. I. Irreducibility of quadratic equations and Nullstellensatz. J. Algebra 200 (1998), 472-516.

[13] O. Kharlampovich and A. Myasnikov. Irreducible affine varieties over a free group. II. Systems in triangular quasi-quadratic form and description of residually free groups. J. Algebra 200 (1998), 517-570.

[14] O. Kharlampovich, A. G. Myasnikov, V. N. Remeslennikov, D. E. Serbin. Subgroups of fully residually free groups: algorithmic problems. In: Group theory, statistics, and cryptography, Contemp. Math., 360 (2004), Amer. Math. Soc., Providence, RI, pp. 63-101.

[15] D. Marker. Model Theory: An Introduction. Springer (2000).

[16] A. G. Myasnikov, V. N. Remeslennikov. Algebraic Geometry over Groups II. Logical Foundations. J. Algebra 234 (2000), 225-276.

[17] A. Ould Houcine. Sur quelques problèmes de plongement dans les groupes. Ph.D. thesis, Université Paris 7, (2003).

[18] A. Ould Houcine. Some properties of finitely generated models. Preprint, (2004).

[19] F. Paulin. Sur la théorie élémentaire des groupes libres (d'après Sela). Astérisque 294 (2004), 363-402.

[20] Z. Sela. Diophantine geometry over groups. I. Makanin-Razborov diagrams. Publ. Math. Inst. Hautes Études Sci, 93 (2001) 31-105. 
[21] Z. Sela. Diophantine Geometry over Groups VIII: The elementary theory of a hyperbolic groups. Preprint.

[22] B.A.F. Wehrfrits. Infinite Linear Groups. Springer-Verlag, New York/Heidelberg/Berlin, 1973.

Abderezak OULD HOUCINE,

Institut Camille Jordan,

Université Claude Bernard Lyon-1,

Bâtiment Braconnier, 21 Avenue Claude Bernard,

69622 Villeurbanne Cedex, France.

ould@math. univ-lyon1.fr 